TRANSACTIONS OF THE

AMERICAN MATHEMATICAL SOCIETY

Volume 360, Number 10, October 2008, Pages 5509-5528

S 0002-9947(08)04619-9

Article electronically published on May 29, 2008

\title{
ROTATION NUMBERS FOR RANDOM DYNAMICAL SYSTEMS ON THE CIRCLE
}

\author{
WEIGU LI AND KENING LU
}

\begin{abstract}
In this paper, we study rotation numbers of random dynamical systems on the circle. We prove the existence of rotation numbers and the continuous dependence of rotation numbers on the systems. As an application, we prove a theorem on analytic conjugacy to a circle rotation.
\end{abstract}

\section{INTRODUCTION}

In this paper, we consider a class of random maps of the circle arising in the study of dynamical systems when randomness or noise is taken into account.

Let $(\Omega, \mathcal{F}, \mathbb{P})$ be a probability space and $\theta$ be a measurable $\mathbb{P}$-measure preserving map on $\Omega$. Let $\psi: S^{1} \times \Omega \rightarrow S^{1}$ be an orientation preserving random map of the circle, where $S^{1}=\mathbb{R} / 2 \pi \mathbb{Z}$. This random map generates a forward random dynamical system

$$
\Psi(n, \cdot, \omega)= \begin{cases}\psi\left(\cdot, \theta^{n-1} \omega\right) \circ \cdots \circ \psi(\cdot, \omega), & n>0, \\ I, & n=0 .\end{cases}
$$

Let $\varphi(x, \omega): \mathbb{R} \times \Omega \rightarrow \mathbb{R}$ be a lift of $\psi$,

$$
\psi\left(e^{i x}, \omega\right)=e^{i \varphi(x, \omega)},
$$

which satisfies

(1) $\varphi(x+2 \pi, \omega)=\phi(x, \omega)+2 \pi$;

(2) $\varphi(x, \omega)$ is monotonic increasing with respect to $x$, i.e.,

$$
\varphi(x, \omega) \geq \varphi(y, \omega), \quad \text { for } x \geq y .
$$

We use $\phi(n, \omega) x$ to denote the random dynamical system generated by $\varphi$. A random map $\varphi$ is called a continuous random map if $\varphi(x, \omega)$ is continuous in $x$ for each fixed $\omega \in \Omega[$

We assume that

(3) $\varphi(x, \cdot) \in L^{1}(\Omega, \mathcal{F}, \mathbb{P})$.

Received by the editors November 24, 2006

2000 Mathematics Subject Classification. Primary 60H15; Secondary 34C35, 58F11, 58F15, $58 \mathrm{~F} 36$.

Key words and phrases. Rotation number, random maps of circle, random differential equations.

This work was partially supported by NSF0200961, NSF0401708, and NSFC10371083 (second author) and NSFC10531010 and NNSF10525104 (first author).

${ }^{1}$ It is enough to assume that $\varphi$ is continuous in $x$ almost surely in $\omega$.

(C)2008 American Mathematical Society 
Let $L^{1}\left(\Omega, M\left(S^{1}\right)\right)$ denote the set of random maps $\varphi(x, \omega)$ of the circle satisfying the above conditions (1), (2), and (3). We introduce a metric in $L^{1}\left(\Omega, M\left(S^{1}\right)\right)$ as follows:

$$
d\left(\phi_{1}, \phi_{2}\right)=\int_{\Omega} \sup _{x \in \mathbb{R}}\left|\phi_{1}-\phi_{2}\right| d \mathbb{P} .
$$

Denote by $L^{1}\left(\Omega, H\left(S^{1}\right)\right)$ the subset of $L^{1}\left(\Omega, M\left(S^{1}\right)\right)$ of the continuous random maps.

Our main results can be summarized as

Theorem A. Let $\varphi \in L^{1}\left(\Omega, M\left(S^{1}\right)\right)$. Then,

(i) Existence of rotation number: There exists a forward $\theta$-invariant set $\tilde{\Omega} \in \mathcal{F}$ of full measure and an $L^{1}$-function $\rho(\cdot): \tilde{\Omega} \rightarrow \mathbb{R}$ such that

$$
\lim _{n \rightarrow \infty} \frac{\phi(n, \omega) x}{2 \pi n}=\rho(\omega), \text { for all } x \in \mathbb{R}, \omega \in \tilde{\Omega},
$$

and $\rho\left(\theta^{n} \omega\right)=\rho(\omega)$, for all $n \in \mathbb{N}, \rho(\omega)$ is constant when $\theta$ is ergodic, and where $\phi(n, \omega) x$ is the random dynamical system generated by $\varphi$.

(ii) Continuous dependence:

$$
\rho: L^{1}\left(\Omega, H\left(S^{1}\right)\right) \rightarrow L^{1}(\Omega, \mathcal{F}, \mathbb{P}): \varphi \mapsto \rho(\omega) .
$$

is continuous

(iii) Compact metric space: If, in addition, $\Omega$ is a compact metric space, $\varphi(x, \omega)$ and $\theta$ are continuous, and $\mathbb{P}$ is the unique $\theta$-invariant probability measure, then

$$
\lim _{n \rightarrow \infty} \frac{\phi(n, \omega) x}{2 \pi n}=\rho, \text { a real constant for all } x \in \mathbb{R} \text { and all } \omega \in \Omega .
$$

(iv) Random ODE on the circle: Analogous results hold for random ODE's on $S^{1}$,

$$
x^{\prime}=f\left(x, \theta^{t} \omega\right) .
$$

Theorem A is an extension of the classical results on rotation numbers of orientation-preserving homeomorphisms of the circle to orientation-preserving random maps of the circle and to random ODE's on the circle. The classical results on rotation numbers can be found in 12 .

As an application of this theorem, we consider the case when $\Omega=\mathbb{R}^{m-1} / 2 \pi \mathbb{Z}$ is the torus of dimension $m-1$ and the dynamical system $\left\{\theta^{n}\right\}_{n \in \mathbb{Z}}$ on $\Omega$ is given by

$$
\theta^{n}: \omega \mapsto \omega+2 n \pi \alpha,
$$

where $\alpha \in \mathbb{R}^{m-1}$ is a given vector. We assume that

$$
\langle\alpha, k\rangle-j \neq 0 \text {, for all } k \in \mathbb{Z}^{m-1} \backslash\{0\}, j \in \mathbb{Z} .
$$

Then, the normalized Lebesgue measure $\mathbb{P}$ is the unique $\theta$-invariant probability measure and $\theta$ is ergodic under $\mathbb{P}$. Let $\varphi(x, \omega)$ be a random map of the circle in $L^{1}\left(\Omega, M\left(S^{1}\right)\right)$. Suppose that $\varphi(\cdot, \cdot)$ is continuous. Then, by Theorem A, the rotation number of $\varphi$ exists and is given by

$$
\begin{aligned}
\rho & =\lim _{n \rightarrow \infty} \frac{\phi(n, \omega) x}{2 \pi n} \\
& =\lim _{n \rightarrow \infty} \frac{1}{2 \pi n} \varphi\left(\cdot, \theta^{n-1} \omega\right) \circ \varphi\left(\cdot, \theta^{n-2} \omega\right) \circ \cdots \circ \varphi(x, \omega), \quad \text { for all } x \in \mathbb{R}, \omega \in \Omega,
\end{aligned}
$$

which is independent of $\omega$ and $x$. 
We denote by $U_{r}$ the strip region in $\mathbb{C}^{m}$,

$$
U_{r}:=\left\{z=\left(z_{1}, z_{2}, \ldots, z_{m}\right) \in \mathbb{C}^{m}:\left|\operatorname{Im} z_{i}\right|<r, i=1,2, \ldots, m\right\},
$$

where $r$ is a positive number. For a holomorphic function $p(z)$ bounded in this region, we define

$$
\|p\|_{r}=\sup _{z \in U_{r}}|p(z)|
$$

For a vector valued analytic function $f(z)=\left(f_{1}(z), f_{2}(z), \ldots, f_{m}(z)\right): U_{r} \rightarrow \mathbb{C}^{m}$, we define

$$
\|f\|_{r}=\max _{1 \leq j \leq m}\left\|f_{j}\right\|_{r}
$$

Consider a perturbation of the circle rotation by $2 \pi \rho$,

$$
\varphi(x, \omega)=x+2 \pi \rho+p(x, \omega) .
$$

We have the following theorem on analytic conjugacy to a circle rotation.

Theorem B. Let $p(x, \omega)$ be analytic in $U_{r}$ and $2 \pi$-periodic in each variable, real on the real axes. Assume

(1) $\varphi(x, \omega)=x+2 \pi \rho+p(x, \omega)$ has the rotation number $\rho$ and

(2) the vector $\mu=(\rho, \alpha)$ is of $(C, \nu)$ type, i.e.,

$$
\left|e^{2 \pi i\langle\mu, k\rangle}-1\right|>\frac{C}{|k|^{\nu}}, \quad|k|:=\left|k_{1}\right|+\left|k_{2}\right|+\cdots+\left|k_{m}\right|
$$

for all nonzero integer vector $k \in \mathbb{Z}^{m}$, where $C$ and $\nu$ are positive constants. Then, there exists $\epsilon>0$ depending only on $C, \nu, r$ and $m$ such that if $\|p\|_{r}<\epsilon$, then the random map $\varphi(\cdot, \omega)$ is analytically conjugate to the circle rotation by the angle $2 \pi \rho$, i.e., there exists an analytical random transformation

$$
H(\cdot, \cdot): \mathbb{R}^{m} / 2 \pi \mathbb{Z} \rightarrow \mathbb{R} / 2 \pi \mathbb{Z}
$$

such that

$$
H(x+2 \pi \rho, \theta \omega)=\varphi(\cdot, \omega) \circ H(x, \omega) .
$$

When $\varphi(x, \omega)$ is independent of $\omega$, Theorem B was first proved by V. Arnold 2, [3]; see also [12. Theorem B is also related to the classical result that a family of analytic homeomorphisms

$$
y \mapsto y+\alpha+p(y), \quad \text { where } \alpha, y \in \mathbb{T}^{n},
$$

for most of $\alpha$, is analytically conjugate to a translation $y \mapsto y+2 \pi \mu$ if $p$ is sufficiently small.

The study of rotation numbers of homeomorphims of the circle goes back to Poincaré and has a rich history. It plays an important role in the investigation of qualitative behavior of various dynamical systems. There is an extensive literature on this subject. We will not try to give an exhaustive list of references, but mention only some related works here. For the classical results such as the Poincaré classification theorem and the Denjoy theorem we refer to Katok and Hasselblatt [12. For the application of rotation numbers to the spectral theory of almost periodic Schrödinger operators, see Johnson and Moser [11]. Recently, Fabbri, Johnson, and Nunez [5, 6], 7] have obtained the results on rotation numbers for non-autonomous linear Hamiltonian Systems. The rotation numbers of asymmetric equations were studied by Feng and Zhang [8]. For the rotation numbers of stochastic ODE's, see 
the book by L. Arnold and the references therein. The existence of rotation numbers in Theorem A is also related to the work by Ruffino [16] on rotation numbers of linear processes in $\mathbb{R}^{2}$. There is a nice survey article by Franks 9] on rotation numbers in dynamics on surfaces and their applications to the description of dynamical systems. For diffeomorphisms of the circle, there are rich results on smooth conjugacy to circle rotations; see the works of Arnold[3], Herman [10, Yoccoz 18, Katznelson and Ornstein [13, Sinai and Khanin 17, and their references therein.

We organize this paper as follows. In Section 2, we prove the main results on rotation numbers for random maps of the circle. Analogous results for random ODE's on the circle are given in Section 3. In Section 4, we apply our main results to almost periodic ordinary differential equations and random compositions of homeomorphisms of the circle. The proof of Theorem B and a more general result are given in Section 5. The proof of Theorem B is based on the standard KAM approach.

\section{Rotation NUmbers of RANDOM MAPS OF THE CIRCLE}

In this section, we prove our main results for orientation preserving random maps of the circle. We formulate properties (i), (ii), and (iii) in Theorem A as the following propositions.

Proposition 2.1 (Existence of rotation numbers). Let $\varphi \in L^{1}\left(\Omega, M\left(S^{1}\right)\right)$. Then, there exists a forward $\theta$-invariant set $\tilde{\Omega} \in \mathcal{F}$ of full measure and a $L^{1}$-function $\rho(\omega): \tilde{\Omega} \rightarrow \mathbb{R}$ such that

$$
\lim _{n \rightarrow \infty} \frac{\phi(n, \omega) x}{2 \pi n}=\rho(\omega), \text { for all } x \in \mathbb{R}, \omega \in \tilde{\Omega},
$$

and $\rho\left(\theta^{n} \omega\right)=\rho(\omega)$ for all $n \in \mathbb{N}, \rho(\omega)$ is constant when $\theta$ is ergodic, and where $\phi(n, \omega) x$ is the random dynamical system generated by $\varphi$.

Proof. First we claim that

$$
\sup _{x \in \mathbb{R}}(\phi(n, \omega) x-x) \leq \inf _{x \in \mathbb{R}}(\phi(n, \omega) x-x)+2 \pi .
$$

Indeed, let $y<x<y+2 \pi$; then

$\phi(n, \omega) y-y-2 \pi \leq \phi(n, \omega) y-x \leq \phi(n, \omega) x-x \leq \phi(n, \omega) y+2 \pi-x \leq \phi(n, \omega) y-y+2 \pi$, which yields (2). For any $x<y$, let $m=[y-x]+1$, where [.] denotes the integer part of the number; then

$$
\phi(n, \omega) x \leq \phi(n, \omega) y \leq \phi(n, \omega) x+2 \pi m .
$$

Thus, if the limit $\lim _{n \rightarrow \infty} \phi(n, \omega) x / n$ exists, it is independent of the point $x$. Let

$$
\Theta(x, \omega):=(\phi(1, \omega) x, \theta \omega)
$$

denote the corresponding skew product map and write $\phi(1, \omega) x=\varphi(x, \omega)=x+$ $h(x, \omega)$. Then, $h(x+2 \pi, \omega)=h(x, \omega)$. Thus, by the definition of $\Theta$, we have

$$
\begin{aligned}
\phi(s+t, \omega)(0) & =\sum_{k=0}^{s+t-1} h \circ \Theta^{k}(0, \omega)=\phi(s, \omega)(0)+\sum_{k=s}^{s+t-1} h \circ \Theta^{k}(0, \omega) \\
& =\phi(s, \omega)(0)+\phi\left(t, \theta^{s} \omega\right) \phi(s, \omega)(0)-\phi(s, \omega)(0) .
\end{aligned}
$$

Hence, by claim (2), we have

(4) $\phi(s, \omega)(0)+\phi\left(t, \theta^{s} \omega\right)(0)-2 \pi \leq \phi(s+t, \omega)(0) \leq \phi(s, \omega)(0)+\phi\left(t, \theta^{s} \omega\right)(0)+2 \pi$. 
Let $f_{n}(\omega)=\phi(n, \omega)(0)+2 \pi, g_{n}(\omega)=-\phi(n, \omega)(0)+2 \pi$; then by inequality (4), we obtain

$$
f_{s+t}(\omega) \leq f_{s}(\omega)+f_{t}\left(\theta^{s} \omega\right), \quad g_{s+t}(\omega) \leq g_{s}(\omega)+g_{t}\left(\theta^{s} \omega\right)
$$

By Kingman's Subadditive Ergodic Theorem, there is a forward invariant set $\tilde{\Omega}$ of full measure and measurable functions $\rho(\omega), \tilde{\rho}(\omega): \tilde{\Omega} \rightarrow \mathbb{R} \cup\{-\infty\}$ with $\rho(\theta \omega)=$ $\rho(\omega), \tilde{\rho}(\theta \omega)=\tilde{\rho}(\omega)$ and $\rho^{+}, \tilde{\rho}^{+} \in L^{1}(\Omega, \mathcal{F}, \mathbb{P})$, where $a^{+}:=\max (0, a)$, such that

$$
\lim _{n \rightarrow \infty} \frac{f_{n}(\omega)}{2 \pi n}=\rho(\omega), \quad \lim _{n \rightarrow \infty} \frac{g_{n}(\omega)}{2 \pi n}=\tilde{\rho}(\omega), \text { for } x \in \mathbb{R}, \omega \in \tilde{\Omega} .
$$

Obviously, $\rho(\omega)=-\tilde{\rho}(\omega)$, which implies that $\rho(\omega) \in L^{1}(\Omega, \mathcal{F}, \mathbb{P})$. When $\theta$ is ergodic, $\rho$ is a constant. This completes the proof of the proposition.

Proposition 2.2 (Continuous dependence of rotation numbers). The mapping

$$
\rho: L^{1}\left(\Omega, H\left(S^{1}\right)\right) \rightarrow L^{1}(\Omega, \mathcal{F}, \mathbb{P}): \varphi \mapsto \rho(\omega)
$$

is continuous

Before we prove this proposition, we review some basic concepts and results on random dynamical systems, which are taken from Arnold [1]. Let $(\Omega, \mathcal{F}, \mathbb{P})$ be a probability space. Let $\mathbb{T}=\mathbb{R}, \mathbb{R}^{+}, \mathbb{Z}$, or $\mathbb{Z}^{+}$. $\mathbb{T}$ is endowed with its Borel $\sigma$-algebra $\mathcal{B}(\mathbb{T})$.

Definition 2.3. A family $\left(\theta^{t}\right)_{t \in \mathbb{T}}$ of mappings from $\Omega$ into itself is called a metric dynamical system if

(1) $(\omega, t) \rightarrow \theta^{t} \omega$ is $\mathcal{F} \otimes \mathcal{B}(\mathbb{T})$ measurable;

(2) $\theta^{0}=i d_{\Omega}$, the identity on $\Omega$, and $\theta^{t+s}=\theta^{t} \circ \theta^{s}$ for all $t, s \in \mathbb{T}$;

(3) $\theta^{t}$ preserves the probability measure $\mathbb{P}$.

Definition 2.4. Let $X$ be a metric space. A map

$$
\phi: \mathbb{T} \times \Omega \times X \rightarrow X, \quad(t, \omega, x) \mapsto \phi(t, \omega, x),
$$

is called a random dynamical system (or a cocycle) over a metric dynamical system $\left(\Omega, \mathcal{F}, \mathbb{P}, \theta^{t}\right)$ if

(1) $\phi$ is $\mathcal{B}(\mathbb{T}) \otimes \mathcal{F} \otimes \mathcal{B}(X)$-measurable.

(2) The map $\phi(t, \omega):=\phi(t, \omega, \cdot): X \rightarrow X$ forms a cocycle over $\theta^{t}$ :

$$
\begin{gathered}
\phi(0, \omega)=I d, \quad \text { for all } \omega \in \Omega, \\
\phi(t+s, \omega)=\phi\left(t, \theta^{s} \omega\right) \circ \phi(s, \omega), \quad \text { for all } t, s \in \mathbb{T}, \quad \omega \in \Omega .
\end{gathered}
$$

A probability measure $\mu$ on $(X \times \Omega, \mathcal{B} \otimes \mathcal{F})$ is said to be an invariant measure for the random dynamical system $\phi$, or $\phi$-invariant, if it satisfies

(i) $\Theta(t) \mu=\mu$ for all $t \in \mathbb{T}$,

(ii) $\pi_{\Omega} \mu=\mathbb{P}$,

where $\Theta(t)(x, \omega)=\left(\phi(t, \omega) x, \theta^{t} \omega\right)$ and $\pi_{\Omega}: X \times \Omega \rightarrow \Omega$ is the projection on $\Omega$.

Let

$\mathcal{P}_{\mathbb{P}}(X \times \Omega):=\{\mu: \mu$ is a probability measure on $(X \times \Omega, \mathcal{B} \otimes \mathcal{F})$

with marginal $\mathbb{P}$ on $(\Omega, \mathcal{F})\}$

and

$$
\mathcal{I}_{\mathbb{P}}(\phi):=\left\{\mu \in \mathcal{P}_{\mathbb{P}}(X \times \Omega): \mu \text { is } \phi \text {-invariant }\right\}
$$


By Theorem 1.5.10 in [1], if $X$ is a compact metric space and $\phi$ is a continuous random dynamical system on $X$, then $\mathcal{I}_{\mathbb{P}}(\phi)$ is not empty.

Suppose that $X$ is a compact metric space. By Proposition 1.4.3 in [1, any probability measure $\mu \in \mathcal{P}_{\mathbb{P}}(X \times \Omega)$ has a $\mathbb{P}$-a.s. unique factorization

$$
\mu(d x, d \omega)=\mu_{\omega}(d x) \mathbb{P}(d \omega),
$$

or equivalently, for all $f \in L_{\mu}^{1}(X \times \Omega)$

$$
\int_{X \times \Omega} f d \mu=\int_{\Omega}\left(\int_{X} f(x, \omega) \mu_{\omega}(d x)\right) \mathbb{P}(d \omega) .
$$

Let $\mathcal{C}_{b}(X)$ denote the Banach space of real-valued bounded continuous functions on $X$, with sup norm $\|f\|_{b}:=\sup _{x \in X}|f(x)|$. We call a function $f: \Omega \rightarrow \mathcal{C}_{b}(X)$ measurable if $(x, \omega) \mapsto f(x, \omega)$ is measurable. Define

$$
L_{\mathbb{P}}^{1}\left(\Omega, \mathcal{C}_{b}(X)\right):=\left\{f: \Omega \rightarrow \mathcal{C}_{b}(X) \text { measurable, }\|f\|:=\int_{\Omega}\|f(\omega, \cdot)\|_{b} d \mathbb{P}<\infty\right\} .
$$

Definition $2.5([1])$. We call the smallest topology in $\mathcal{P}_{\mathbb{P}}(X \times \Omega)$, which makes $\mu \mapsto \mu(f)$ continuous for each $f \in L_{\mathbb{P}}^{1}\left(\Omega, \mathcal{C}_{b}(X)\right)$, the weak convergence on $\mathcal{P}_{\mathbb{P}}(X \times \Omega)$. A sequence $\left\{\mu_{i}\right\}$ converges in the topology to $\mu$ if $\mu_{i}(f) \rightarrow \mu(f)$ for each $f \in L_{\mathbb{P}}^{1}\left(\Omega, \mathcal{C}_{b}(X)\right)$.

Let $X$ be a metric space and $L_{\mathbb{P}}^{1}\left(\Omega, \mathcal{L} i p_{b}(X)\right)$ denote the subset of $L_{\mathbb{P}}^{1}\left(\Omega, \mathcal{C}_{b}(X)\right)$ such that

$$
\operatorname{Lip}(f):=\left|\sup _{x \neq y} \frac{|f(x, \cdot)-f(y, \cdot)|}{|x-y|}\right|_{L_{\mathbb{P}}^{\infty}(\Omega)}<\infty .
$$

Lemma 2.6. Let $X$ be a metric space and $\mu, \nu \in \mathcal{P}_{\mathbb{P}}(X \times \Omega)$. Then

$$
\mu=\nu \Leftrightarrow \int f d \mu=\int f d \nu, \text { for all } f \in L_{\mathbb{P}}^{1}\left(\Omega, \mathcal{L} i p_{b}(X)\right) .
$$

The proof of this lemma follows the same lines as Lemma 1.5.4 in [1].

Lemma 2.7. Let $X$ be a compact metric space. Let $\phi_{i}(t, \omega) x$ be a sequence of continuous random dynamical systems over the metric dynamical system $\left\{\theta^{t}\right\}_{t \in \mathbb{T}}$ on $X$ and $\mu_{i}$ be an invariant measure of $\phi_{i}$. If $\phi_{i}$ converges to a continuous random dynamical system $\phi(t, \omega) x$ in the following sense,

$$
\int_{\Omega} \sup _{x \in X} d\left(\phi_{i}(t, \omega) x, \phi(t, \omega) x\right) d \mathbb{P} \rightarrow 0, \text { for any given } t \in \mathbb{T} \cap[0,1],
$$

then every limit point of $\mu_{i}$ for $i \rightarrow \infty$ in the topology of weak convergence is an invariant measure for $\phi$.

Proof. Assume that $\mu_{i} \rightarrow \mu$ in the topology of weak convergence. Since $\mathcal{P}_{\mathbb{P}}(X \times \Omega)$ is compact by Theorem 1.5.10 in [1], $\mu \in \mathcal{P}_{\mathbb{P}}(X \times \Omega)$. Next, we show that $\mu$ is invariant for $\phi$. Denote by $\Theta_{i}(t)$ and $\Theta(t)$ the corresponding skew-product of $\phi_{i}$ and $\phi$, respectively. For fixed $t \in \mathbb{T} \cap[0,1]$ and for any given $f \in L_{\mathbb{P}}^{1}\left(\Omega, \mathcal{L} i p_{b}(X)\right)$,

$$
\lim _{i \rightarrow \infty} \mid\left(\Theta_{i}(t) \mu_{i}(f)-\Theta(t) \mu_{i}(f) \mid \leq \lim _{i \rightarrow \infty} \operatorname{Lip}(f) \int_{\Omega} \sup _{x \in X} d\left(\phi_{i}(t, \omega) x, \phi(t, \omega) x\right) d \mathbb{P}=0 .\right.
$$

Therefore,

$$
\Theta(t) \mu(f)=\lim _{i \rightarrow \infty} \Theta(t) \mu_{i}(f)=\lim _{i \rightarrow \infty} \Theta_{i}(t) \mu_{i}(f)=\lim _{i \rightarrow \infty} \mu_{i}(f)=\mu(f) .
$$

This implies by Lemma 2.6 that $\Theta(t) \mu=\mu$. The proof is complete. 
Proof of Proposition 2.2. Denote by $\rho_{\varphi}(\omega)$ the rotation number of $\varphi$. First, we note that the rotation number $\rho$ is monotonic with respect to $\varphi$, i.e., $\rho_{\varphi} \geq \rho_{\tilde{\varphi}}$ for $\varphi \geq \tilde{\varphi}$. If the proposition is not true, there exists a sequence of mappings $\varphi_{i}(x, \omega)=x+h_{i}(x, \omega) \in L^{1}\left(\Omega, H\left(S^{1}\right)\right)$ with $\varphi_{i} \rightarrow \varphi=x+h(x, \omega)$ in $L^{1}\left(\Omega, H\left(S^{1}\right)\right)$ such that

$$
\lim _{i \rightarrow \infty} \int_{\Omega}\left|\rho_{\varphi_{i}}(\omega)-\rho_{\varphi}(\omega)\right| d \mathbb{P} \neq 0
$$

Let

$$
\varphi_{i}^{+}=x+h_{i}^{+}(x, \omega):=\max \left\{\varphi, \varphi_{i}\right\}, \phi_{i}^{-}=x+h_{i}^{-}(x, \omega):=\min \left\{\varphi, \varphi_{i}\right\} ;
$$

then $\left\|\varphi_{i}^{+}-\varphi_{i}^{-}\right\| \rightarrow 0$. Let $\nu_{i}^{+}$and $\nu_{i}^{-}$be the invariant probability measures of $\phi_{i}^{+}$ and $\phi_{i}^{-}$, respectively. Then by the fact that $\mathcal{P}_{\mathbb{P}}(X \times \Omega)$ is compact and by Lemma 2.7. we can assume that they converge to some invariant measures $\nu^{+}$and $\nu^{-}$of $\phi$ in the weak topology, respectively. Finally, by using Fubini's Theorem, (3), and Birkhoff's Ergodic Theorem, we have

$$
\begin{aligned}
& \lim _{i \rightarrow \infty} \int_{\Omega}\left|\rho_{\varphi_{i}}(\omega)-\rho_{\varphi}(\omega)\right| d \mathbb{P} \\
& \leq \lim _{i \rightarrow \infty} \int_{\Omega}\left(\rho_{\varphi_{i}^{+}}(\omega)-\rho_{\varphi_{i}^{-}}(\omega)\right) d \mathbb{P} \\
& =\lim _{i \rightarrow \infty}\left(\int_{X \times \Omega} \rho_{\phi_{i}^{+}}(\omega) d \nu_{i}^{+}-\int_{X \times \Omega} \rho_{\phi_{i}^{-}}(\omega) d \nu_{i}^{-}\right) \\
& =\lim _{i \rightarrow \infty}\left(\int_{X \times \Omega} h_{i}^{+} d \nu_{i}^{+}-\int_{X \times \Omega} h_{i}^{-} d \nu_{i}^{-}\right) \\
& =\lim _{i \rightarrow \infty}\left(\int_{X \times \Omega}\left(h_{i}^{+}-h\right) d \nu_{i}^{+}-\int_{X \times \Omega}\left(h_{i}^{-}-h\right) d \nu_{i}^{-}+\int_{X \times \Omega} h d \nu_{i}^{+}-\int_{X \times \Omega} h d \nu_{i}^{-}\right) \\
& \leq \lim _{i \rightarrow \infty}\left(\left\|h-h_{i}^{+}\right\|+\left\|h-h_{i}^{-}\right\|\right)+\lim _{i \rightarrow \infty}\left(\int_{X \times \Omega} h d \nu_{i}^{+}-\int_{X \times \Omega} h d \nu_{i}^{-}\right) \\
& =\int_{X \times \Omega} h d \nu^{+}-\int_{X \times \Omega} h d \nu^{-} \\
& =\int_{\Omega} \rho_{\varphi} d \mathbb{P}-\int_{\Omega} \rho_{\varphi} d \mathbb{P}=0,
\end{aligned}
$$

which contradicts (5). This completes the proof of Proposition 2.2 .

Proposition 2.8 (Compact metric space). Let $\varphi \in L^{1}\left(\Omega, M\left(S^{1}\right)\right)$. If $\Omega$ is a compact metric space, $\varphi(x, \omega)$ and $\theta$ are continuous, and $\mathbb{P}$ is the unique $\theta$-invariant probability measure, then

$$
\lim _{n \rightarrow \infty} \frac{\phi(n, \omega) x}{2 \pi n}=\rho, \text { a real constant for all } x \in \mathbb{R} \text {, all } \omega \in \Omega .
$$

Proof. Since $\Omega$ is compact, on the one hand, according to Krylov and Bogolyubov's Theorem, the skew product map $\Theta(x, \omega): S^{1} \times \Omega \rightarrow S^{1} \times \Omega$ possesses at least one invariant probability measure. On the other hand, by the assumption that $\mathbb{P}$ is the unique $\theta$-invariant probability measure, we have that $\theta$ is ergodic and $\pi_{\Omega} \mu=\mathbb{P}$ for any invariant probability measure $\mu$ of $\Theta$, where $\pi_{\Omega}: S^{1} \times \Omega \rightarrow \Omega, \pi_{\Omega}(x, \omega)=\omega$, is the projection onto $\Omega$. Therefore, using (3) for any invariant probability measure 
$\mu$ of $\Theta$, we have

$$
\lim _{n \rightarrow \infty} \frac{\phi(n, \omega) x}{2 \pi n}=\lim _{n \rightarrow \infty} \frac{1}{2 n \pi} \sum_{k=0}^{n-1} h \circ \Theta^{k}(x, \omega)=\rho, \mu \text {-a.s., }
$$

where $\rho$ is a real number. Thus, by Birkhoff's Ergodic Theorem, we obtain

$$
\int_{S^{1} \times \Omega} h d \mu=\lim _{n \rightarrow \infty} \int_{S^{1} \times \Omega} \frac{1}{n} \sum_{k=0}^{n-1} h \circ \Theta^{k} d \mu=\int_{S^{1} \times \Omega} \lim _{n \rightarrow \infty} \frac{1}{n} \sum_{k=0}^{n-1} h \circ \Theta^{k} d \mu=2 \pi \rho .
$$

To complete the proof of Proposition 2.8, we need the following lemma.

Lemma 2.9. Let $B$ be a compact metric space or a compact Hausdorff separable space and $\left\{\Theta^{t}\right\}_{t \in \mathbb{T}}$ be a continuous dynamical system on B. Suppose that $G$ is a continuous function on $B$ such that

$$
\int_{B} G d \mu=0
$$

for all invariant probability measures $\mu$ of $\Theta$. Then

$$
\begin{gathered}
\lim _{n \rightarrow \infty} \frac{1}{n} \sum_{k=0}^{n-1} G \circ \Theta^{k} u=0, \mathbb{T} \text { discrete, } \\
\lim _{T \rightarrow \infty} \frac{1}{T} \int_{0}^{T} G \circ \Theta^{t} u d t=0, \mathbb{T} \text { continuous, }
\end{gathered}
$$

for all $u \in B$, and the convergence is uniform.

Applying this lemma to the continuous function $G=h-\rho$, we have

$$
\lim _{n \rightarrow \infty} \frac{1}{2 n \pi} \sum_{k=0}^{n-1} h \circ \Theta^{k}(x, \omega)=\rho,
$$

for all $(x, \omega) \in S^{1} \times \Omega$. This completes the proof of Proposition 2.8 ,

Proof of Lemma 2.9. Since $B$ is a compact metric space or a compact Hausdorff separable space, the space $C(B)$ of the continuous functions on $B$ is separable. So we can find a dense linear subspace $D$ generated by a countable set of functions. If the statement is not true, then for some function $G \in C(B)$, we can choose $D$ so that $G \in D$ and select $n_{i} \in \mathbb{N}$ (or $T_{i} \in \mathbb{R}$, respectively) and $u_{i} \in B$ such that $n_{i}\left(\operatorname{or}_{i}\right) \rightarrow \infty$ and

$$
\lim _{n_{i} \rightarrow \infty} \frac{1}{n_{i}} \sum_{k=0}^{n_{i}-1} G \circ \Theta^{k} u_{i}=\delta \neq 0, \mathbb{T} \text { discrete, }
$$

or respectively,

$$
\lim _{T_{i} \rightarrow \infty} \frac{1}{T_{i}} \int_{0}^{T_{i}} G \circ \Theta^{t} u_{i} d t=\delta \neq 0, \mathbb{T} \text { continuous. }
$$

We may assume that $u_{i} \rightarrow \tilde{u}$. Using the Cantor diagonal process we can choose a subsequence, which we still denote by $n_{i}$ (or $T_{i}$ ) and $u_{i}$, such that

$$
\lim _{n_{i} \rightarrow \infty} \frac{1}{n_{i}} \sum_{k=0}^{n_{i}-1} H \circ \Theta^{k} u_{i} \quad \text { exists }
$$


or, respectively,

$$
\lim _{T_{i} \rightarrow \infty} \frac{1}{T_{i}} \int_{0}^{T_{i}} H \circ \Theta^{t} u_{i} d t \quad \text { exists }
$$

for all $H \in D$. This limit defines a linear functional $l=l(H), H \in D$. Since $l$ is bounded with norm 1 , it can be extended uniquely to a bounded positive linear functional on $C(B)$. Obviously, $l\left(H \circ \Theta^{t}\right)=l(H)$. By the Riesz representation theorem, $l$ defines an invariant probability measure $\mu$ on $B$. By our assumption

$$
\int_{B} G d \mu=l(G)=\delta \neq 0
$$

which is a contradiction. The proof of the lemma is complete.

\section{RotATION NUMBERS OF RANDOM DIFFERENTIAL EQUATIONS ON THE CIRCLE}

In this section, we establish the analogous results of Section 3 for random differential equations on the circle.

Let $\left(\Omega, \mathcal{F}, \mathbb{P},\left(\theta^{t}\right)_{t \in \mathbb{R}}\right)$ be a metric dynamical system. Let $C\left(S^{1}\right)$ be the Banach space of real-valued bounded continuous functions on $S^{1}=\mathbb{R} / 2 \pi \mathbb{Z}$. Let $L^{1}\left(\Omega, C_{L}\left(S^{1}\right)\right)$ denote the set of the functions $f(\cdot, \omega)$ which is Lipschitz continuous with respect to $x$ for each fixed $\omega$ and let

$$
M(\omega):=\max \left\{\sup _{x \in \mathbb{R}}|f(x, \omega)|, \sup _{x \neq y} \frac{|f(x, \omega)-f(y, \omega)|}{|x-y|}\right\} \in L^{1}(\Omega, \mathcal{F}, \mathbb{P}) .
$$

In $L^{1}\left(\Omega, C_{L}\left(S^{1}\right)\right)$, we introduce the norm

$$
\|f\|=\int_{\Omega} \sup _{x}|f(x, \omega)| d \mathbb{P} .
$$

Consider the following random ordinary differential equation on the circle $S^{1}=$ $\mathbb{R} / 2 \pi \mathbb{Z}$ :

$$
\dot{x}=f\left(x, \theta^{t} \omega\right) .
$$

Let $x\left(t, x_{0}, \omega\right)$ denote the solution with the initial value $x=x_{0}$ for $t=0$. Assume that $f \in L^{1}\left(\Omega, C_{L}\left(S^{1}\right)\right)$. Then, by Theorem 2.2.6 in [1], equation (7) with $x\left(0, x_{0}, \omega\right)=x_{0}$ has a unique global solution almost surely in $\omega$.

Theorem 3.1. Let $f \in L^{1}\left(\Omega, C_{L}\left(S^{1}\right)\right)$. Then,

(i) there exists a $\theta^{t}$-invariant set $\tilde{\Omega} \in \mathcal{F}$ of full measure and a $L^{1}$-function $\rho(\omega): \tilde{\Omega} \rightarrow \mathbb{R}$ such that

$$
\begin{aligned}
& \lim _{T \rightarrow \infty} \frac{x\left(T, x_{0}, \omega\right)-x_{0}}{T} \\
& \quad=\lim _{T \rightarrow \infty} \frac{1}{T} \int_{0}^{T} f\left(x\left(t, x_{0}, \omega\right), \theta^{t} \omega\right) d t=\rho(\omega), \text { for all } x_{0} \in \mathbb{R}, \omega \in \tilde{\Omega},
\end{aligned}
$$

and $\rho\left(\theta^{t} \omega\right)=\rho(\omega)$ for all $t \in \mathbb{R}$, and $\rho(\omega)$ is constant when $\theta^{t}$ is ergodic;

(ii) the map

$$
\rho: L_{\mathbb{P}}^{1}\left(\Omega, \mathcal{L} i p_{b}\left(S^{1}\right)\right) \rightarrow L^{1}(\Omega, \mathcal{F}, \mathbb{P}): f(x, \omega) \mapsto \rho(\omega)
$$

is continuous; 
(iii) if, in addition, $\Omega$ is a compact metric space, $\theta$ and $f(x, \omega)$ are continuous with $M(\omega)$ bounded, and $\mathbb{P}$ is the unique $\theta$-invariant probability measure, then there exists $\rho \in \mathbb{R}$ such that

$$
\lim _{T \rightarrow \infty} \frac{x\left(T, x_{0}, \omega\right)-x_{0}}{T}=\lim _{T \rightarrow \infty} \frac{1}{T} \int_{0}^{T} f\left(x\left(t, x_{0}, \omega\right), \theta^{t} \omega\right) d t=\rho \in \mathbb{R}
$$

for all $x_{0} \in \mathbb{R}$, and all $\omega \in \Omega$.

Proof. We first prove (i). Since $M(\omega) \in L^{1}$, we have that the solution $x\left(t, x_{0}, \omega\right)$ of the initial value problem exists globally for almost all $\omega \in \Omega$. We note that the function $x\left(t, x_{0}, \omega\right)-x_{0}$ is $2 \pi$-periodic and $x\left(t, x_{0}, \omega\right)$ is increasing with respect to $x_{0}$. Therefore, the limit in (i), if exists, is independent of $x_{0}$. Let

$$
\Omega_{1}:=\left\{\omega \in \Omega: \lim _{n \rightarrow \infty} \frac{1}{n} \int_{n}^{n+1} M\left(\theta^{t} \omega\right) d t=0\right\}
$$

From Lemma 2.2.5 and Proposition 4.1.3 in [1] it follows that $\Omega_{1}$ is a $\theta^{t}$-invariant set of full measure. Let $\tilde{\Omega}$ denote the set of $\omega \in \Omega_{1}$ such that the limit in (i) exists. Then, $\tilde{\Omega}$ is a $\theta^{t}$-invariant set and the limit is $\theta$-invariant. Next, we show that it has a full measure. By Proposition 2.1, there exists an invariant set $\Omega_{2}$ of full measure such that

$$
\lim _{\mathbb{N} \ni n \rightarrow \infty} \frac{x\left(n, x_{0}, \omega\right)-x_{0}}{n}
$$

exists for $\omega \in \Omega_{2}$. For $\omega \in \Omega_{1} \cap \Omega_{2}$, we have

$$
\lim _{T \rightarrow \infty} \frac{1}{T} \int_{0}^{T} f\left(x\left(t, x_{0}, \omega\right), \theta_{t} \omega\right) d t=\lim _{T \rightarrow \infty} \frac{1}{[T]} \int_{0}^{[T]} f\left(x\left(t, x_{0}, \omega\right), \theta^{t} \omega\right) d t .
$$

Since $\tilde{\Omega} \supset \Omega_{1} \cap \Omega_{2}$ and $\Omega_{1}$ and $\Omega_{2}$ have a full measure, $\tilde{\Omega}$ has a full measure.

Next, we show that (ii) holds. Denote by $x_{f}\left(t, x_{0}, \omega\right)$ the solution of equation (77) with the initial value $x=x_{0}$ at $t=0$. Then, by Gronwall's inequality, for $f, g \in L_{\mathbb{P}}^{1}\left(\Omega, \mathcal{L} i p_{b}\left(S^{1}\right)\right)$ we have

$$
\left\|x_{f}(t, \cdot, \cdot)-x_{g}(t, \cdot, \cdot)\right\| \leq t\|f-g\| \exp (t(\operatorname{Lip}(f))), \text { for any given } t \in \mathbb{R},
$$

which together with Proposition 2.2 gives (ii) in the theorem.

Finally, we show (iii). Let $\Theta^{t}\left(x_{0}, \omega\right)=\left(x\left(t, x_{0}, \omega\right), \theta_{t} \omega\right)$, the corresponding skew product flow. Since $\mathbb{P}$ is the unique $\theta$-invariant probability measure, we have that $\theta$ is ergodic and $\pi_{\Omega} \mu=\mathbb{P}$ for any invariant probability measure $\mu$ of $\Theta$. Therefore, by (i) there exists a constant $\rho$ such that for any invariant probability measure $\mu$ of $\Theta$,

$$
\rho=\lim _{T \rightarrow \infty} \frac{1}{T} \int_{0}^{T} f \circ \Theta^{t}\left(x_{0}, \omega\right) d t, \text { a.s., } \mu \text {. }
$$


Thus, by using Lebesgue's Dominated Convergence Theorem, Fubini's Theorem, and Birkhoff's Ergodic Theorem, we have

$$
\begin{aligned}
\rho & =\int \rho d \mu \\
& =\int\left(\lim _{T \rightarrow \infty} \frac{1}{T} \int_{0}^{T} f \circ \Theta^{t} d t\right) d \mu \\
& =\lim _{T \rightarrow \infty} \int \frac{1}{T} \int_{0}^{T} f \circ \Theta^{t} d t d \mu \\
& =\lim _{T \rightarrow \infty} \frac{1}{T} \int_{0}^{T} \int f \circ \Theta^{t} d \mu d t \\
& =\int f d \mu .
\end{aligned}
$$

Applying Lemma 2.9 for the continuous time to the function $f-\rho$, we obtain (3). This completes the proof of this theorem.

\section{Applications}

In this section, we apply our main results to almost periodic differential equations. Let $f(x, u):(\mathbb{R} / 2 \pi \mathbb{Z}) \times \mathbb{R}^{d} \rightarrow \mathbb{R}$ be a continuous function and $f(\cdot, u)$ be Lipschitz continuous with respect to $x$ for each fixed $u$. Assume that

$$
M(u)=\lim _{\delta \rightarrow 0} \sup _{0<|x-y|<\delta} \frac{|f(x, u)-f(y, u)|}{|x-y|}
$$

is a locally bounded measurable function in $u \in \mathbb{R}^{d}$. Suppose that $u(t): \mathbb{R} \rightarrow \mathbb{R}^{d}$ is an almost periodic function. Consider the following almost periodic time-depending differential equation on $S^{1}$ :

$$
\dot{x}=f(x, u(t)) .
$$

Denote by $x\left(t, x_{0}\right)$ the solution of equation (8) with the initial value $x\left(0, x_{0}\right)=x_{0}$.

Theorem 4.1. The rotation number $\rho$ defined by the limit

$$
\rho:=\lim _{T \rightarrow \infty} \frac{x\left(T, x_{0}\right)-x_{0}}{T}=\lim _{T \rightarrow \infty} \frac{1}{T} \int_{0}^{T} f\left(x\left(t, x_{0}\right), u(t)\right) d t
$$

exists and is independent of $x_{0}$.

Proof. Let $H(u):=\{u(\cdot+t): t \in \mathbb{R})$ be the hull of $u$, i.e., the set of all translates of $u$. Then the closure $\overline{H(f)}$ of $H(f)$ is compact and consists of almost periodic functions, where the closure is taken in the uniform topology. $\overline{H(f)}$ has the structure of a compact Abelian Polish group $G$ with unit $e=u$. The group operation * is defined as follows: For $g=u(\cdot+t)$ and $h=u(\cdot+s), g * h=u(\cdot+t+s)$, while for $g=\lim u\left(\cdot+t_{n}\right)$ and $h=\lim u\left(\cdot+s_{n}\right), g * h=\lim u\left(\cdot+t_{n}+s_{n}\right)$.

Associated to the almost periodic function $u$, we have the following canonical metric dynamical system. Let $\mathbb{T}=\mathbb{R}, \Omega:=G=\overline{H(f)}$, and $\mathcal{F}$ be the Borel $\sigma$ algebra of $G$. The metric dynamical system is given by the translation of $\omega$ by $t$, $\theta_{t} \omega: \omega(\cdot+t)$. Note that $(t, \omega) \mapsto \theta_{t} \omega$ is continuous. The normalized Haar measure 
$\mathbb{P}$ of $G$ is the unique $\theta$-invariant probability. Under $\mathbb{P}, \theta$ is ergodic. We define a continuous function $F$ on $(\mathbb{R} / 2 \pi \mathbb{Z}) \times \Omega$ as

$$
F(x, \omega):=f(x, \omega(0)) .
$$

Now consider the random ordinary differential equation on the circle:

$$
\dot{x}=F\left(x, \theta_{t} \omega\right) .
$$

Then by Theorem 3.1, for each $\omega \in \Omega$, the rotation number of equation (9) exists and is independent of $\omega \in \Omega$. Obviously, for $\omega=u, F\left(x, \theta_{t} \omega\right)=f(x, u(t))$. This completes the proof.

Example 1. Let $A(x, u): \mathbb{R}^{2} \times \mathbb{R}^{d} \rightarrow \mathbb{R}^{2}$ be continuous and be Lipschitz continuous in $x$ with a locally bounded measurable Lipschitz constant in $u$. Assume that $A$ is positive homogeneous with respect to $x$, i.e.,

$$
A(\lambda x, u)=\lambda A(x, u), \quad \text { for } \lambda \geq 0 .
$$

Let $u(t): \mathbb{R} \rightarrow \mathbb{R}^{d}$ be an almost periodic function. Consider an almost periodic time-depending system in $\mathbb{R}^{2}$ as

$$
\dot{x}=A(x, u(t)), \quad x=\left(x_{1}, x_{2}\right)^{T} \in \mathbb{R}^{2} .
$$

In polar coordinates $r=\left(x_{1}^{2}+x_{2}^{2}\right)^{1 / 2}, \alpha=\arctan \left(x_{2} / x_{1}\right)$, the system (10) is written as

$$
\dot{r}=\langle A(w, u(t)), w\rangle r, \quad \dot{\alpha}=\langle A(w, u(t)), v\rangle,
$$

where $w=(\cos \alpha, \sin \alpha)^{T}, v=(-\sin \alpha, \cos \alpha)^{T}$. Denote by $\left(r\left(t, r_{0}, \alpha_{0}\right), \alpha\left(t, \alpha_{0}\right)\right)$ the solution of the system with the initial value condition $\left(r\left(0, r_{0}, \alpha_{0}\right), \alpha\left(0, \alpha_{0}\right)\right)=$ $\left(r_{0}, \alpha_{0}\right)$. Then the rotation number of the system (10) is defined to be the linear growth rate of the angular component, i.e., by

$$
\rho:=\lim _{T \rightarrow \infty} \frac{\alpha\left(t, \alpha_{0}\right)-\alpha_{0}}{T}=\lim _{T \rightarrow \infty} \frac{1}{T} \int_{0}^{T}\langle A(w, u(t)), v\rangle d t .
$$

By Theorem 4.1, the rotation number exists and is independent of the initial value.

Example 2. Let $f_{1}, f_{2}, \ldots, f_{k}$ be the orientation preserving homeomorphisms of the circle with the same invariant probability measure $\mu_{x}$ and let $\left(p_{1}, p_{2}, \ldots, p_{k}\right)$ be a probability vector with non-zero entries (i.e., $p_{i}>0$ for each $i$ and $\sum_{i=1}^{k} p_{i}=1$ ). Assume that $f_{i}$ has the rotation number $\rho_{i}$. Let $\left(K, 2^{K}, \mu\right)$ denote the probability space where $K=\{1,2, \ldots, k\}$ and the point $i$ has measure $p_{i}$. Let

$$
(\Omega, \mathcal{F}, \mathbb{P})=\prod_{1}^{\infty}\left(K, 2^{K}, \mu\right) .
$$

We write points of $\Omega$ in the form $\omega=\left(\omega_{1}, \omega_{2}, \ldots\right), \omega_{i} \in K$, and define $\theta: \Omega \rightarrow \Omega$ by

$$
\theta\left(\omega_{1}, \omega_{2}, \ldots\right)=\left(\omega_{2}, \omega_{3}, \ldots\right) .
$$

Then, $\theta$ is $\mathbb{P}$ measure-preserving. We define an orientation preserving random map of the circle as $\varphi(x, \omega)=f_{\omega_{1}}$. Then the product measure $\mu_{x} \times \mathbb{P}$ is the invariant measure of the dynamical system $\left.\Theta^{n}(x, \omega)=\left(\phi(n, \omega) x, \theta^{n} \omega\right)\right)$, where

$$
\phi(n, \omega) x=f_{\omega_{n}} \circ \cdots \circ f_{\omega_{2}} \circ f_{\omega_{1}}(x)
$$

is the random dynamical system over $\theta$ generated by $\varphi(x, \omega)$. 
Let $f_{i}(x)=x+h_{i}(x), \varphi(x, \omega)=x+h(x, \omega)$. By formula (6), the rotation number of $\phi$ is

$$
\begin{aligned}
\rho & =\frac{1}{2 \pi} \int_{S^{1} \times \Omega} h d \mathbb{P} d \mu_{x} \\
& =\frac{1}{2 \pi} \int_{S^{1}} \sum_{i=1}^{k} p_{i} h_{i}(x) d \mu_{x} \\
& =\frac{1}{2 \pi} \sum_{i=1}^{k} p_{i} \lim _{n \rightarrow \infty} \int_{S^{1}} \frac{1}{n} \sum_{m=0}^{n-1} h_{i} \circ f_{i}^{m} d \mu_{x} \\
& =\frac{1}{2 \pi} \sum_{i=1}^{k} p_{i} \int_{S^{1}} \lim _{n \rightarrow \infty} \frac{1}{n} \sum_{m=0}^{n-1} h_{i} \circ f_{i}^{m} d \mu_{x} \\
& =\frac{1}{2 \pi} \sum_{i=1}^{k} p_{i} \int_{S^{1}} 2 \pi \rho_{i} d \mu_{x} \\
& =\sum_{i=1}^{k} p_{i} \rho_{i} .
\end{aligned}
$$

This example shows that the rotation number of random compositions of the orientation preserving homeomorphisms of the circle, $f_{1}, \ldots, f_{k}$, is the probability weighted average of the rotation numbers of them.

\section{Analytical conjugacy to a Circle rotation}

In this section, we study the problem of analytic conjugacy to a circle rotation. We first review a Diophantine condition.

Definition 5.1. We say that $\mu \in \mathbb{R}^{m}$ is a vector of type $(C, \nu)$ if

$$
\left|e^{2 \pi i\langle\mu, k\rangle}-1\right|>\frac{C}{|k|^{\nu}}, \quad|k|:=\left|k_{1}\right|+\left|k_{2}\right|+\cdots+\left|k_{m}\right|
$$

for all non-zero integer vector $k \in \mathbb{Z}^{m}$.

The next lemma gives that almost all of vectors $\mu \in \mathbb{R}^{m}$ satisfy (11).

Lemma 5.2. Let $\nu>m$ be a constant. For almost every real vector $\mu \in \mathbb{R}^{m}$, there exists $C=C(\mu, \nu)>0$ such that the inequality (11) holds for all non-zero integer vectors $k \in \mathbb{Z}^{m}$.

Proof. The proof of this lemma follows from the standard argument. First we claim that for almost every vector $\mu \in \mathbb{R}^{m}$ there exists $C=C(\mu, \nu)>0$ such that

$$
|\langle k, \mu\rangle-q|>\frac{C}{|k|^{\nu}}
$$

for all $k \in \mathbb{Z}^{m} \backslash\{0\}$ and $q \in \mathbb{Z}$. Indeed, we fix a ball in $\mathbb{R}^{m}$ and estimate the measure of the set of $\mu$ in it which does not satisfy the inequality (12). Let

$$
L_{k, q}=\left\{\mu \in \mathbb{R}^{m}:\langle k, \mu\rangle-q=0\right\}
$$

denote the resonance plane. The inequality

$$
|\langle k, \mu\rangle-q| \leq \frac{C}{|k|^{\nu}}
$$


determines a neighborhood of width not greater than $C_{1} C /|k|^{\nu+1}$ of the resonance plane. Therefore, the measure of the part of this neighborhood which is contained in the ball does not exceed $C_{2} C /|k|^{\nu+1}$. Summing over $k$ with fixed $|k|$, we obtain that the measure is not more than $C_{3} C /|k|^{\nu-m+2}$. Summing over $q$ with fixed $|k|$ such that distance between $L_{k, q}$ and the ball is less than 1, we obtain that the measure is bounded by $C_{4} C /|k|^{\nu-m+1}$. Summing over $|k|$, we have that the measure is bounded by $C_{5}(\nu) C<\infty$. Consequently, the set of $\mu$ in the ball is covered by the sets of arbitrarily small measure. Hence, such a set has measure zero.

The proof of this lemma follows this claim since the distance of $\langle k, \mu\rangle$ from the closest integer is bounded from below by $C /|k|^{\nu}$ and a chord of the unit circle is not shorter than the length of the small arc subtended by it divided by $\frac{\pi}{2}$. This completes the proof of the lemma.

We now consider a class of random maps of the circle over an $m-1$ dimensional torus. Let $\Omega=\mathbb{R}^{m-1} / 2 \pi \mathbb{Z}$ be the torus of dimension $m-1$. Consider the metric dynamical system $\left\{\theta^{n}\right\}_{n \in \mathbb{Z}}$ on $\Omega$ given by

$$
\theta^{n}: \omega \mapsto \omega+2 n \pi \alpha,
$$

where $\alpha \in \mathbb{R}^{m-1}$ is a given vector. We assume that

$$
\langle\alpha, k\rangle-j \neq 0, \text { for all } k \in \mathbb{Z}^{m-1} \backslash\{0\}, j \in \mathbb{Z} .
$$

Then, the normalized Lebesgue measure $\mathbb{P}$ is the unique $\theta$-invariant probability measure and $\theta$ is ergodic under $\mathbb{P}$. Let $\varphi(x, \omega):(\mathbb{R} / 2 \pi \mathbb{Z} \times \Omega) \rightarrow \mathbb{R} / 2 \pi \mathbb{Z}$ be an orientation preserving random map of the circle over $\theta$. Suppose that $\varphi(\cdot, \cdot)$ is continuous. Then, by Theorem A, the rotation number of $\varphi$ exists and is given by

$$
\begin{aligned}
\rho & =\lim _{n \rightarrow \infty} \frac{\phi(n, \omega) x}{2 \pi n} \\
& =\lim _{n \rightarrow \infty} \frac{1}{2 \pi n} \varphi\left(\cdot, \theta^{n-1} \omega\right) \circ \varphi\left(\cdot, \theta^{n-2} \omega\right) \circ \cdots \circ \varphi(x, \omega), \quad \text { for all } x \in \mathbb{R}, \omega \in \Omega,
\end{aligned}
$$

which is independent of $\omega$ and $x$.

Consider a perturbation of the circle rotation by $2 \pi \rho$ :

$$
\varphi(x, \omega)=x+2 \pi \rho+p(x, \omega),
$$

where $p(x, \omega)$ is a holomorphic function defined on the strip $U_{r}$ which was introduced in the Introduction.

We have the following theorem on analytic conjugacy to a circle rotation.

Theorem 5.3. Let $p(x, \omega)$ be analytic in $U_{r}$ and $2 \pi$-periodic in each variable, and real on the real axes. Assume

(1) $\varphi(x, \omega)=x+2 \pi \rho+p(x, \omega)$ has the rotation number $\rho$ and

(2) the vector $\mu=(\rho, \alpha)$ is of $(C, \nu)$ type, i.e.,

$$
\left|e^{2 \pi i\langle\mu, k\rangle}-1\right|>\frac{C}{|k|^{\nu}}, \quad|k|:=\left|k_{1}\right|+\left|k_{2}\right|+\cdots+\left|k_{m}\right|
$$

for all non-zero integer vectors $k \in \mathbb{Z}^{m}$, where $c$ and $\nu$ are positive constants.

Then, there exists $\epsilon>0$ depending only on $C, \nu, r$ and $m$ such that if $\|p\|_{r}<\epsilon$, then the random map $\varphi(\cdot, \omega)$ is analytically conjugate to the circle rotation by the angle $2 \pi \rho$, i.e., there exists an analytical random transformation

$$
H(\cdot, \cdot): \mathbb{R}^{m} / 2 \pi \mathbb{Z} \rightarrow \mathbb{R} / 2 \pi \mathbb{Z}
$$


such that

$$
H(x+2 \pi \rho, \theta \omega)=\varphi(\cdot, \omega) \circ H(x, \omega) .
$$

Instead of proving Theorem 5.3 , we will prove a more general result which gives Theorem 5.3 as its special case. We consider a mapping

$$
\Phi(z): \mathbb{R}^{m} / 2 \pi \mathbb{Z} \rightarrow \mathbb{R}^{m} / 2 \pi \mathbb{Z}
$$

Definition 5.4. We say that the mapping $\Phi(z)$ has a rotation vector $\mu \in \mathbb{R}^{m}$, if

$$
\lim _{n \rightarrow \infty} \frac{1}{n} \Phi^{n}(z)=2 \pi \mu
$$

for all $z \in \mathbb{R}^{m} / 2 \pi \mathbb{Z}$.

Consider a perturbation of a vector rotation:

$$
\Phi(z)=z+2 \pi \mu+p(z): \mathbb{R}^{m} / 2 \pi \mathbb{Z} \rightarrow \mathbb{R}^{m} / 2 \pi \mathbb{Z} .
$$

We have the following result.

Theorem 5.5. Let $p(z)$ be analytic in $U_{r}$ and $2 \pi$-periodic function in each variable, and real on the real axes. Assume

(1) $\Phi(z)=z+2 \pi \mu+p(z): \mathbb{R}^{m} / 2 \pi \mathbb{Z} \rightarrow \mathbb{R}^{m} / 2 \pi \mathbb{Z}$ has a rotation vector $\mu$ and

(2) the rotation vector $\mu$ is of $(C, \nu)$ type for positive constants $C$ and $\nu$.

Then, there exists $\epsilon>0$ depending only on $C, \nu, r$ and $m$ such that if $\|p\|_{r}<\epsilon$, then $\Phi(z)$ is analytically conjugate to the rotation $R_{\mu}: z \mapsto z+2 \pi \mu$, i.e., there exists an analytic transformation

$$
H(z)=z+h(z): \mathbb{R}^{m} / 2 \pi \mathbb{Z} \rightarrow \mathbb{R}^{m} / 2 \pi \mathbb{Z},
$$

where $h(z)$ is a $2 \pi$-periodic function in each variable, such that

$$
H \circ R_{\mu}=\Phi \circ H \text {. }
$$

The proof of this theorem is based on the classic KAM approach. We write $H(z)$ in the form $H(z)=z+h(z)$, where $h(z)$ is $2 \pi$-periodic in each variable. Then substituting it into conjugate equation (14), we obtain the functional equation for $h$,

$$
h(z+2 \pi \mu)-h(z)=p(z+h(z))
$$

The first approximation of this equation is the so called homological equation for $h$,

$$
h(z+2 \pi \mu)-h(z)=p(z) .
$$

Obviously, the homological equation is not solvable if the mean value of $p(z)$ is nonzero. We expand the given function $p$ and the unknown function $h$ in the Fourier series:

$$
p(z)=\sum_{k \in \mathbb{Z}^{m} \backslash\{0\}} p_{k} e^{i\langle k, z\rangle}, \quad h(z)=\sum_{k \in \mathbb{Z}^{m} \backslash\{0\}} h_{k} e^{i\langle k, z\rangle} .
$$

Plugging them into (15) and comparing the coefficients of $e^{i\langle k, z\rangle}$, we have

$$
h_{k}=\frac{p_{k}}{e^{2 \pi i\langle\mu, k\rangle}-1}, \quad k \in \mathbb{Z}^{m} \backslash\{0\} .
$$

The idea of the proof of Theorem 5.5 is the following. We solve the homological equation (15) with the right-hand side $\tilde{p}(z)=p(z)-p_{0}$, where $p_{0}$ is the mean value of the function $p(z)$. Denote the solution by $h^{0}$. Let $H_{0}(z)=z+h^{0}(z)$. Set $\Phi_{1}=H_{0}^{-1} \circ \Phi \circ H_{0}$ and define a function $p^{1}(z)$ by the relation

$$
\Phi_{1}(z)=z+2 \pi \mu+p^{1}(z) .
$$


The next approximation is constructed in the same way. Beginning with $\Phi_{1}$ in place of $\Phi$, we solve the corresponding homological equation for $h^{1}$ and let $H_{1}=z+h^{1}(z)$. The transformation $H_{1}$ converts $\Phi_{1}$ into

$$
\Phi_{2}=H_{1}^{-1} \circ \Phi_{1} \circ H_{1} .
$$

Repeating this procedure, we have a sequence of the transformation $H_{n}$. Let

$$
\mathbf{H}_{n}=H_{0} \circ H_{1} \circ \cdots \circ H_{n-1} \text {. }
$$

Then

$$
\Phi_{n}=\mathbf{H}_{n}^{-1} \circ \Phi \circ \mathbf{H}_{n} .
$$

Finally, we will prove that $\lim _{n \rightarrow \infty} \Phi_{n}=z+2 \pi \mu$.

Before proving the theorem, we first introduce several lemmas which we will need later.

Lemma 5.6. Let $f: \mathbb{R}^{m} \rightarrow \mathbb{R}$ be $2 \pi$-periodic in each component, and be analytic in the strip $U_{r}$ and continuous in the closure of this strip. Assume that $\|f\|_{r} \leq M$. Then its Fourier coefficients satisfy

$$
\left|f_{k}\right| \leq M e^{-|k| r}
$$

Proof. For $k=\left(k_{1}, k_{2}, \ldots, k_{m}\right) \in \mathbb{Z}^{m}$, we define $u=\left(u_{1}, u_{2}, \ldots, u_{m}\right) \in \mathbb{Z}^{m}$ as $u_{j}=-\operatorname{sgn}\left(k_{j}\right), j=1,2, \ldots, m$. Then we have

$$
f_{k}=\frac{1}{(2 \pi)^{m}} \int_{\mathbb{T}^{m}} e^{-i\langle k, z\rangle} f(z) d z=\frac{1}{(2 \pi)^{m}} \int_{\mathbb{T}^{m}} e^{-i\langle k, z\rangle-|k| r} f(z+i r u) d z .
$$

Hence,

$$
\left|f_{k}\right| \leq \frac{1}{(2 \pi)^{m}} \int_{\mathbb{T}^{m}}\left|e^{-i\langle k, z\rangle-|k| r} f(z+i r u)\right| d z \leq M e^{-|k| r} .
$$

Lemma 5.7. If $\left|f_{k}\right| \leq M e^{-|k| r}$, then the function $f=\sum f_{k} e^{i\langle k, z\rangle}$ is analytic in the strip $U_{r}$ and $\|f\|_{r-\delta} \leq 8\left(\frac{4 m-4}{e}\right)^{m-1} M \delta^{-m}$, where $\delta<\min \{1, r\}$.

Proof. The proof follows from the following computation:

$$
\begin{aligned}
\|f\|_{r-\delta} & \leq \sum\left|f_{k}\right|\left|e^{i\langle k, z\rangle}\right| \leq \sum M e^{-|k| r} e^{|k|(r-\delta)} \\
& =M \sum e^{-|k| \delta}=M \sum_{l=0}^{\infty} \frac{2^{m}(l+m-1) !}{l !(m-1) !} e^{-l \delta} \\
& \leq 2^{m} M \sum_{l=0}^{\infty}(l+1)^{m-1} e^{-l \delta} \leq 2^{m} M e^{\delta}\left(\frac{2 m-2}{e \delta}\right)^{m-1} \sum_{l=0}^{\infty} e^{-\delta l / 2} \\
& \leq M e^{\delta}\left(\frac{4 m-4}{e}\right)^{m-1} \frac{1}{\delta^{m-1}} \frac{1}{1-e^{-\delta / 2}} \leq 8 M\left(\frac{4 m-4}{e}\right)^{m-1} \delta^{-m} .
\end{aligned}
$$

Lemma 5.8. Let $p(z): U_{r} \rightarrow \mathbb{C}^{m}$ be a $2 \pi$-periodic analytic function with mean value 0 . Let $h(z)$ be the solution of the homological equation (15). Then, there exists a constant $\lambda=\lambda(C, \nu, m)>0$ such that if $\mu$ is of type $(C, \nu)$, then for any $r<\frac{1}{2}$ and any $\delta>0$ smaller than $r$, we have $\|h\|_{r-\delta} \leq\|a\|_{r} \delta^{-\lambda}$.

Proof. Let $M=\|p\|_{r}, p(z)=\left(p_{1}(z), p_{2}(z), \ldots, p_{m}(z)\right)$, and $h(z)=\left(h_{1}(z), h_{2}(z)\right.$, $\left.\ldots, h_{m}(z)\right)$. We write

$$
p_{j}(z)=\sum_{k \in \mathbb{Z}^{m} \backslash\{0\}} p_{k}^{j} e^{i\langle k, z\rangle}, \quad h_{j}(z)=\sum_{k \in \mathbb{Z}^{m} \backslash\{0\}} h_{k}^{j} e^{i\langle k, z\rangle}, j=1,2, \ldots, m .
$$


By Lemma [5.6] we have $\left|p_{k}^{j}\right| \leq M e^{-|k| r}$. Since $\mu$ is of type $(C, \nu)$, using (16) we have

$$
\begin{aligned}
\left|h_{k}^{j}\right| & \leq|k|^{\nu} M e^{-|k| r} / C \leq M C^{-1}|k|^{\nu} e^{-\delta|k| / 2} e^{-|k|(r-\delta / 2)} \\
& \leq M C^{-1}\left(\frac{\nu}{e}\right)^{\nu}\left(\frac{\delta}{2}\right)^{-\nu} e^{-|k|(r-\delta / 2)}
\end{aligned}
$$

By Lemma 5.7 .

$$
\|h\|_{r-\delta}=\max _{1 \leq j \leq m}\left\|h_{j}\right\|_{r-\delta} \leq 8 M C^{-1}\left(\frac{\nu}{e}\right)^{\nu}\left(\frac{\delta}{2}\right)^{-\nu}\left(\frac{4 m-4}{e}\right)^{m-1}\left(\frac{\delta}{2}\right)^{-m} \leq M \delta^{-\lambda},
$$

for $\lambda$ sufficiently large.

The next two lemmas are obvious.

Lemma 5.9. Let $H(z)=z+h(z): \mathbb{R}^{m} / 2 \pi \mathbb{Z} \rightarrow \mathbb{R}^{m} / 2 \pi \mathbb{Z}$ be a homeomorphism, where $h$ is $2 \pi$-periodic in each component of $z$. Suppose that the mapping $\Phi$ : $\mathbb{R}^{m} / 2 \pi \mathbb{Z} \rightarrow \mathbb{R}^{m} / 2 \pi \mathbb{Z}$ has the rotation vector $\mu \in \mathbb{R}^{m} ;$ then the mapping $H^{-1} \circ \Phi \circ H$ has also the rotation vector $\mu$.

Lemma 5.10. Suppose that the homeomorphism

$$
\Phi(z)=z+2 \pi \mu+p(z): \mathbb{R}^{m} / 2 \pi \mathbb{Z} \rightarrow \mathbb{R}^{m} / 2 \pi \mathbb{Z}
$$

has the rotation vector $\mu$, where $p$ is $2 \pi$-periodic in each component of $z$. Then each component $p_{j}(z)$ of $p(z)$ vanishes at some point.

The next one is our main lemma for proving Theorem 5.5 .

Lemma 5.11. There exist positive constants $\tau, \gamma$ depending only on $C, \nu$ and $m$ such that for every $\delta$ in the interval $(0, r)$, where $r<1 / 2$, we have

$$
\left\|p^{1}\right\|_{r-\delta} \leq\|p\|_{r}^{2} \delta^{-\gamma} \text {, provided that }\|p\|_{r} \leq \delta^{\tau} .
$$

Proof. We first show that if $\tau$ is large enough, then $A_{1}$ is well defined and analytic in the strip $U_{r-\delta}$.

Let $M=\|p\|_{r}$ and assume $M \leq \delta^{\tau}$. Then, the mean $p_{0}$ of $p$ satisfies $\left\|p_{0}\right\| \leq M$ and $\|\tilde{p}\|_{r}=\left\|p-p_{0}\right\|_{r} \leq 2 M$. By Lemma 5.8, for any $0<\alpha<r$, we have $\left\|h^{0}\right\|_{r-\alpha} \leq$ $2 M \alpha^{-\lambda}$, which implies

$$
\left\|D h^{0}\right\|_{r-2 \alpha} \leq 2 m M \alpha^{-\lambda-1}
$$

Let $\alpha=\delta / 8$. Choosing $\tau$ is sufficiently large, we obtain

$$
\|p\|_{r}<\alpha, \quad\left\|h^{0}\right\|_{r-\alpha}<\alpha, \quad\left\|D h^{0}\right\|_{r-2 \alpha}<\alpha .
$$

Therefore, $H_{0}(z)=z+h(z)$ is a diffeomorphism on $U_{r-2 \alpha}$ and its image contains $U_{r-3 \alpha}$. Since $H_{0} U_{r-\delta} \subset U_{r-\delta+\alpha}, \Phi \circ H_{0} U_{r-\delta} \subset U_{r-\delta+2 \alpha} \subset U_{r-3 \alpha}$. The inverse $H_{0}^{-1}$ is defined on $\Phi \circ H_{0} U_{r-\delta}$. Hence, the mapping $\Phi_{1}=H_{0}^{-1} \circ \Phi \circ H_{0}$ is well defined and analytic on $U_{r-\delta}$

Next, we estimate the function $p^{1}$. Since $H_{0} \circ \Phi_{1}=\Phi \circ H_{0}$, we have

$$
z+2 \pi \mu+p^{1}(z)+h^{0}\left(z+2 \pi \mu+p^{1}(z)\right)=z+h^{0}(z)+2 \pi \mu+p\left(z+h^{0}(z)\right)
$$

or

$$
\begin{aligned}
p^{1}(z) & =p\left(z+h^{0}(z)\right)+h^{0}(z)-h^{0}\left(z+2 \pi \mu+p^{1}(z)\right) \\
& =p\left(z+h^{0}(z)\right)-p(z)+h^{0}(z+2 \pi \mu)-h^{0}\left(z+2 \pi \mu+p^{1}(z)\right)+p_{0} .
\end{aligned}
$$


Thus,

$$
\begin{aligned}
\left\|p^{1}(z)\right\|_{r-\delta} \leq \| & \left\|\left(z+h^{0}(z)\right)-p(z)\right\|_{r-\delta} \\
& +\left\|h^{0}(z+2 \pi \mu)-h^{0}\left(z+2 \pi \mu+p^{1}(z)\right)\right\|_{r-\delta}+\left\|p_{0}\right\| .
\end{aligned}
$$

By the mean value theorem and the Cauchy inequality, we have

$$
\left\|p\left(z+h^{0}(z)\right)-p(z)\right\|_{r-\delta} \leq\|D p\|_{r-\alpha}\left\|h^{0}\right\|_{r-\delta} \leq \frac{M}{\alpha} M \delta^{-\lambda}=8 M^{2} \delta^{-\lambda-1} \leq M^{2} \delta^{-\kappa},
$$

where the constant $\kappa$ depends only on $\lambda$, i.e., hence only on $C, \nu$ and $m$. Similarly, we have

$\left\|h^{0}(z+2 \pi \mu)-h^{0}\left(z+2 \pi \mu+p^{1}(z)\right)\right\|_{r-\delta} \leq\left\|D h^{0}\right\|_{r-\alpha}\left\|p^{1}\right\|_{r-\delta} \leq 2 m M \alpha^{-\lambda-1}\left\|p^{1}\right\|_{r-\delta}$.

By Lemma [5.9, the rotation vector of $\Phi_{1}$ is $\mu$. Thus, from Lemma 5.10, every component $p_{j}^{1}(z)$ of $p^{1}(z)$ vanishes at some real point, say $z_{0}^{j} \in \mathbb{R}^{m}, j=1,2, \ldots, m$. Let $p_{0}=\left(p_{1}^{0}, p_{2}^{0}, \ldots, p_{m}^{0}\right)$. Then

$$
p_{j}^{0}=p_{j}\left(z_{0}^{j}\right)-p_{j}\left(z_{0}^{j}+h^{0}\left(z_{0}^{j}\right)\right)+h_{j}^{0}\left(z_{0}^{j}+2 \pi \mu+p^{1}\left(z_{0}^{j}\right)\right)-h_{j}^{0}\left(z_{0}^{j}+2 \pi \mu\right) .
$$

Hence,

$$
\begin{aligned}
\left\|p_{0}\right\| & =\max _{1 \leq j \leq m}\left|p_{j}^{0}\right| \\
& \leq \max _{1 \leq j \leq m}\left\{\left|p_{j}\left(z_{0}^{j}\right)-p_{j}\left(z_{0}^{j}+h^{0}\left(z_{0}^{j}\right)\right)\right|+\left|h_{j}^{0}\left(z_{0}^{j}+2 \pi \mu+p^{1}\left(z_{0}^{j}\right)\right)-h_{j}^{0}\left(z_{0}^{j}+2 \pi \mu\right)\right|\right\} \\
& \leq\left\|p(z)-p\left(z+h^{0}(z)\right)\right\|_{r-\delta}+\left\|h^{0}\left(z+2 \pi \mu+p^{1}(z)\right)-h^{0}(z+2 \pi \mu)\right\|_{r-\delta} \\
& \leq M^{2} \delta^{-u}+2 m M \alpha^{-\lambda-1}\left\|p^{1}\right\|_{r-\delta} .
\end{aligned}
$$

Combining (19), (20), and (21) with (18), we obtain

$$
\begin{aligned}
\left\|p^{1}\right\|_{r-\delta} & \leq 2 M^{2} \delta^{-\kappa}\left(1-4 m M \alpha^{-\lambda-1}\right)^{-1} \leq 2 M^{2} \delta^{-\kappa}\left(1-4 m \delta^{\tau}(\delta / 8)^{-\lambda-1}\right)^{-1} \\
& =2 M^{2} \delta^{-\kappa}\left(1-m 2^{3 \lambda+5} \delta^{\tau-\lambda-1}\right)^{-1} \leq 4 M^{2} \delta^{-\kappa} \leq M^{2} \delta^{-\gamma}
\end{aligned}
$$

provided that $\tau$ is sufficiently large. Here $\gamma=\kappa+2$.

Proof of Theorem [5.5. Let $\delta_{0} \leq \frac{1}{2}, \delta_{n}=\delta_{n-1}^{3 / 2}$, and fix $\delta_{0}$ small enough such that $\sum_{n=0}^{\infty} \delta_{n}<\frac{r}{2}$. Set $r_{0}=r, r_{n}=r_{n-1}-\delta_{n-1}, M_{n}=\delta_{n}^{N}$, where $N=\max \{\tau, 2 \lambda\}$. Assume that $\|p\|_{r} \leq M_{0}$; we claim that

$$
\left\|p^{n}\right\|_{r_{n}} \leq M_{n}, \quad n \geq 0 .
$$

We prove (22) by induction. Assume that for $n=k$, inequality (22) holds. Then by Lemma 5.11 .

$$
\left\|p^{k+1}\right\|_{r_{k+1}} \leq M_{k}^{2} \delta_{k}^{-\gamma}=\delta_{k}^{2 N-\gamma} \leq \delta_{k}^{3 N / 2}=\delta_{k+1}^{N}=M_{k+1} .
$$

Next, we prove the convergence of the composition $\mathbf{H}_{n}=H_{0} \circ H_{1} \circ \cdots \circ H_{n-1}$. First, by Lemma 5.8 and (17), we have that for $\tau$ sufficiently large the diffeomorphism $H_{0}$ is analytic in $U_{r}$ and satisfies $\left\|h^{0}\right\|_{r_{1}}<\delta_{0},\left\|D h^{0}\right\|_{r_{1}}<\delta_{0}$. By induction, we have that for any $n \geq 0$,

$$
\left\|h^{n-1}\right\|_{r_{n}}<\delta_{n-1},\left\|D h^{n-1}\right\|_{r_{n}}<\delta_{n-1}
$$


and $\mathbf{H}_{n}$ is analytic in $U_{r_{n}}$. Therefore, the derivative of $\mathbf{H}_{n}$ satisfies

$$
0<C_{-}:=\prod_{k=0}^{\infty}\left(1-\delta_{k}\right) \leq\left\|D \mathbf{H}_{n}\right\|_{r_{n}} \leq \prod_{k=0}^{\infty}\left(1+\delta_{k}\right):=C_{+} .
$$

Hence $\mathbf{H}_{n}$ is a diffeomorphism. Note that

$$
\left\|\mathbf{H}_{n}-\mathbf{H}_{n+1}\right\|_{r / 2} \leq C_{+}\left\|h^{n}\right\|_{r / 2} \leq C_{+} \delta_{n} .
$$

So, the sequence $\mathbf{H}_{n}$ converges on $U_{r / 2}$. Let

$$
\mathbf{H}=\left.\lim _{n \rightarrow \infty} \mathbf{H}_{n}\right|_{U_{r / 2}} .
$$

Then,

$$
\mathbf{H} \circ R_{\mu}=\lim _{n \rightarrow \infty} \mathbf{H}_{n} \circ \Phi_{n}=\lim _{n \rightarrow \infty} \Phi \circ \mathbf{H}_{n}=\Phi \circ \mathbf{H} .
$$

This completes the proof of this theorem.

\section{ACKNowledgement}

We would like to thank Jiangong You for his comments and suggestion on random compositions of homeomorphisms of the circle. The first author would also like to thank the Department of Mathematic at Brigham Young University for their support and hospitality during the fall of 2004 when this paper was written.

\section{REFERENCES}

[1] L. Arnold. Random Dynamical Systems. Springer, New York, 1998. MR1723992 (2002m:37087)

[2] V. I. Arnold. Geometric Methods in the Theory of Ordinary Differential Equations. SpringerVerlag, New York, 1983. MR695786 (84d:58023)

[3] V. I. Arnold. Small denominators I. On the mapping of a circle into itself. Izv. Akad. Nauk. Math., 25 (1961), 21-86. MR0140699 (25:4113)

[4] S.-N. Chow, K. Lu, and Y.-Q. Shen. Normal form and linearization for quasiperiodic systems. Trans. Amer. Math. Soc., 331 (1992), 361-376. MR.1076612 (92g:34048)

[5] R. Fabbri, R. Johnson, and C. Núñez. Rotation number for non-autonomous linear Hamiltonian systems. II. The Floquet coefficients. Z. Angew. Math. Phys., 54 (2003), 652-676. MR.1994030 (2005f:37115)

[6] R. Fabbri, R. Johnson, and C. Núñez. Rotation number for non-autonomous linear Hamiltonian systems. I. Basic properties. Z. Angew. Math. Phys., 54 (2003), 484-502. MR2048667 (2005f:37114)

[7] R. Fabbri, R. Johnson, and C. Núñez. Disconjugacy and the rotation number for linear, nonautonomous Hamiltonian systems. Ann. Mat. Pura Appl., 185 (2006), S3-S21. MR2187752 (2007e:37012)

[8] H. Feng and M. Zhang. Optimal estimates on rotation number of almost periodic systems. Z. Angew. Math. Phys., 57 (2006), 183-204. MR2214068(2006k:37110)

[9] J. Franks. Rotation numbers and instability sets. Bull. Amer. Math. Soc., 40 (2003), 263279. MR:1978565 (2004h:37063)

[10] M. R. Herman. Sur la conjugaison differentielle des difféomorphismes du cercle á des rotations. Publ. Math. I.H.E.S., 49(1979), 5-234. MR538680(81h:58039)

[11] R. Johnson and J. Moser. The rotation number for almost periodic potentials. Comm. Math. Phys., 84 (1982), 403-438. MR667409 (83h:34018)

[12] A. Katok and B. Hasselblatt. Introduction to the modern theory of dynamical systems. With a supplementary chapter by Katok and Leonardo Mendoza. Encyclopedia of Mathematics and its Applications, 54. Cambridge University Press, Cambridge, 1995. MR1326374 (96c:58055)

[13] Y. Katznelson and D. Ornstein. The differentiability of the conjugation of certain diffeomorphism of the circle. Ergod. Th. and Dyn. Sys., 9 (1989), 643-680. MR1036902 (91i:58121) 
[14] J. K. Moser. A rapidly covergent iteration method and nonlinear differential equations II. Ann. Scuo. Norm. Sup. Pisa., 20 (1966), 499-535. MR0206461(34:6280)

[15] H. Poincaré. Sur le probléme des trois corps et les équations de la dynamique Acta Math., 13 (1890), 1-270.

[16] P. R. C. Ruffino. A sampling theorem for rotation numbers of linear processes in $\mathbb{R}^{2}$. Random operators and Stochastic Equations, 8 (2000), 175-188. MR1765876 (2001e:37009)

[17] Ya. G. Sinai and K. M. Khanin. Smoothness of conjugacies of diffeomorphisms of the circle with rotations. Russ. Math. Surv., 44 (1989), 69-99. MR997684(90i:58183)

[18] J.-C. Yoccoz. Conjugaison differentielle des difféomorphismes du cercle dont le nombre de rotation vérifie une condition Diophantienne. Ann. Sci. Ec. Norm. Sup., 17 (1984), 333-361. MR7777374 (86j:58086)

School of Mathematics, Peking University, Beijing 100871, People's Republic of CHINA

E-mail address: weigu@math.pku.edu.cn

Department of Mathematics, Brigham Young University, Provo, Utah 84602

E-mail address: klu@math. byu.edu 